\title{
Algorithms for fast and precise computation of the normal integral
}

\author{
ALFRED L. BROPHY \\ Behavioral Science Associates, West Chester, Pennsylvania \\ and \\ D. LOUIS WOOD \\ University of Arkansas at Little Rock, Little Rock, Arkansas
}

\begin{abstract}
Five highly accurate algorithms that evaluate the normal distribution function are presented. The algorithms, which are based on either numerical integration or series expansions, are explicated, and applications of Simpson's rule are discussed. Computer programs that implement the algorithms were compared with respect to accuracy and speed. The programs attain from 11- to 15-decimal-place accuracy in finding one-tailed areas of the normal curve, and most execute very quickly. Recommendations are made for selection of algorithms and programs to approximate the normal distribution.
\end{abstract}

Highly accurate computation of the value of the normal integral can be achieved with numerical integration (Cyvin, 1964; Smits, 1981; Wood, 1985, 1987) and with polynomial and rational functions, continued fractions, and series expansions (Johnson \& Kotz, 1970, chap. 13; Kennedy \& Gentle, 1980, chap. 5). In this paper, we review five highly accurate normal curve algorithms, three of which are based on numerical integration, and two on series expansions. We also present, explicate, and compare programs that enact these algorithms, and we describe their performance characteristics. In addition, we explore the efficacy of Simpson's rule in finding normal curve areas with 10- and 15-decimal-place accuracy.

The accuracy of numerical integration is a function of the number of points at which the integral is evaluated. In routines applying Simpson's rule to evaluate the normal integral in the interval $(0, z)$, where $z$ is a standard normal deviate, Smits (1981) used 1,000 segments to attain 10-decimal-place accuracy, and Wood (1985) used 52 segments to attain 6-decimal-place accuracy. In two more versatile programs that compute the integral between any two $z$ values, Wood (1987) used varying numbers of segments to attain from 6-to 12-decimal-place accuracy, and he reported that the programs could be adapted to attain 15-decimal-place accuracy. The number of segments necessary to attain a given level of accuracy can be found empirically or by formula in developing such computer routines. The required number of segments varies with both the desired accuracy and the absolute magnitude of $z$.

Two methods can be used to determine an appropriate number of segments at run time for the input $z$ and for a predetermined or user-specified accuracy level. The first method, which Cyvin (1964) and Wood (1987) employed,

Address correspondence to Alfred L. Brophy, Behavioral Science Associates, P.O. Box 748, West Chester, PA 19381. uses an inequality that provides either the maximum possible error that can occur given a specified number of segments into which $f(z)$ is partitioned, or, following algebraic rearrangement, the number of segments required to mathematically guarantee any specified level of accuracy. The first version of the inequality follows, cited from Anton (1981, p. 534).

$$
\mid \text { error } \mid \leq \frac{M(b-a)^{5}}{180 N^{4}}
$$

The second version takes the following form:

$$
N \leq \sqrt[4]{\frac{M(b-a)^{5}}{180 \mid \text { error } \mid}} .
$$

In these inequalities, $N$ is the number of segments into which an interval of $f(z)$ is divided, and $M$ is the maximum absolute value of $f^{\text {iv }}(z)$ within that interval; $b$ is the upper bound, and $a$ is the lower bound. Values of $f^{\text {iv }}(z)$ can be obtained from Pearson and Hartley (1972, Table 2) or by evaluating the derivative directly as shown below.

$$
f^{\mathrm{iv}}(z)=(2 \pi)^{-1 / 2} e^{-z^{2} / 2}\left(z^{4}-6 z^{2}+3\right) .
$$

The maximum value of $f^{\text {iv }}(z)$ is 1.196827 , occurring at $z=0$.

Study of Inequality 2 provides the keys to efficient numerical integration of $f(z)$ by Simpson's rule. Specifically, $N$ is minimized (and a computer program therefore runs most quickly) when the range of numerical integration (i.e., $b-a)$ is short and the fourth derivative of $f(z)$ is small. $N$ is especially reduced when $(b-a)<1$, because fractions become smaller when raised to powers larger than 1, and when $M<1$. Parenthetically, Simpson's rule requires that $N$ be an even integer; therefore, the value of $N$ is increased to the smallest even integer that is greater than or equal to $N$ as calculated with Inequality 2 . 
The second method of determining the number of segments $(n)$ applies Simpson's rule repeatedly, beginning with $n=2$ and doubling $n$ with each iteration until the difference between two successive results is less than the acceptable error $(r)$. An efficient version of this method reuses previous calculations and newly computes only $(n-1) / 2$ of the points otherwise needed at each iteration. Although the method appears relatively crude, it functions with a substantially lower $n$ than the first method when $(b-a)$ is large, thus reducing both program execution time and roundoff error. For example, with $a=0, b=6$, and $r=10^{-9}$, the first method, using Inequality 2 , establishes $N \leq 478$, but $n$ is only 32 with the second method. In using the first method, Wood (1987) avoided high $N$ when $z$ is large by evaluating the interval $(z, \infty)$ rather than $(0, z)$. (He set $\infty$ equal to an even larger $z$ value, beyond which the area is negligible relative to the precision of the computations.)

\section{ZSIMPIT}

ZSIMPIT (see Appendix A) is a BASIC program that uses the second method to determine $n$. The program requires input of $z$ and $r$; it returns the area $(A)$ in the interval $(0, z)$, the one-tailed probability $(p)$ calculated as $(.5-A), n$, the execution time, and an estimate of the error in $A$ based on the difference between the last two approximations of the integral. The estimated error is frequently larger than the true error, but it is not a bound, and it is sometimes smaller than the true error.

ZSIMPIT could be refined by adding a correction involving derivatives at the ends of the integral or by using Richardson's extrapolation. These techniques would result in lower $n$ for a given $r$, as well as a better estimate of the error. Adaptive programming, which determines an appropriate-and possibly different-number of segments for various subranges within the interval being estimated (Davis \& Rabinowitz, 1975, chap. 6), might be used to reduce total $n$, but that technique, which involves more complex coding, would have little advantage with the wellbehaved normal distribution function. In light of the accuracy and speed of the remaining algorithms, replacement rather than refinement of ZSIMPIT seems more appropriate.

\section{ZQUICK}

ZQUICK (see Appendix B) is an extension of the Simpson's rule algorithms introduced and subsequently elaborated and expanded by Wood $(1985,1987)$. It is an improvement over those routines because it is faster by far and because it always returns normal curve areas with 10 accurate decimals. The substantial increases in both speed and accuracy were obtained through dividing the interval $(0,6.5)$ into 27 subintervals. The area beyond each subinterval, named $A R E A$, was determined using an expanded version of NORMLCV.EXT (Wood, 1987) and was written into the program, as was the $N$ value for each subinterval. When run, the algorithm identifies the subinterval into which a user-entered $z$ fits and then employs Simpson's rule to calculate the area between that $z$ and the upper bound of the subinterval containing it. That area plus $A R E A$ is the one-tailed $p$ associated with $z$. This technique restricts the range of numerical integration to a value smaller than 1 and thus enables accuracy to be obtained quickly, as does the fact that $f^{\text {iv }}(z)<1$ when $|z|=$ 0.26165 . Although the algorithm used in ZQUICK is longer than that cited by Wood (1987), the program still consists of only 58 lines, of which 13 effect the screen display.

When run, ZQUICK requests that $z$ be input; it returns $p, A, 2 A, 2 p$ (the two-tailed probability), and the execution time.

\section{ZQUICK.EXT}

In order to examine the performance of short-range Simpson's rule programming with extremely high precision, we developed an extended version of ZQUICK, named ZQUICK.EXT (see Appendix C), that seeks 15place accuracy. The program functions as does ZQUICK, but with the following exceptions. When the user inputs $z$ and the program identifies the subinterval into which that $z$ falls, $N$ is determined in one of two ways. In six of the subintervals, the maximum value of $f^{\text {iv }}(z)$ is written into the program; in the other subintervals, $M$ is calculated by substituting the user-entered $z$ in Equation 3 . $M$ is next substituted in Inequality 2 , and $N$ is determined. Then, as in ZQUICK, the Simpson's rule subroutine is engaged, and the results are printed out.

The program does deliver its target accuracy. However, its length is 67 lines, and it is slow, requiring a maximum of $40.7 \mathrm{sec}$ when run in GW-BASICA/D 3.2 on an IBM PC microcomputer with $z=1 \mathrm{E}-16$.

\section{ZSTRECOK}

ZSTRECOK (see Appendix D) is an algorithm that combines a simple modification of Strecok's (1968) approximation of the error function with an approximation for large values of $z$ from Peizer and Pratt (1968). Strecok's method was the basis of Moran's (1980) approximation of the normal distribution, which led to very accurate, compact computer routines (Brophy, 1983). Strecok's method computes the error function as

$$
\begin{aligned}
\operatorname{erf}(y) & =2 / \pi^{1 / 2} \int_{0}^{y} e^{-x^{2}} d x \\
& \approx 2 / \pi\left[(y / 5)+\sum_{n=1}^{37} n^{-1} e^{-(n / 5)^{2}} \sin (2 n y / 5)\right]
\end{aligned}
$$

Strecok gave another form of the approximation that avoids 37 sine calculations but that requires storage of a like number of coefficients. Because of the availability of intrinsic high-precision, reasonably fast sine functions and the brevity of a computer routine implementing the original form of the approximation, ZSTRECOK uses the original form. Strecok's approximation is adapted to estimate the normal integral by the relationship

$$
A(z)=(2 \pi)^{-1 / 2} \int_{0}^{z} e^{-x^{2} / 2} d x=\operatorname{erf}\left(z / 2^{1 / 2}\right) / 2 .
$$

Although Equation 4 uses 37 terms, we found that 29 terms produce equivalent results with 16-digit arithmetic. 
ZSTRECOK requires input of $z$; it returns $A, p$, and the execution time.

\section{ZKERCOOK}

ZKERCOOK (see Appendix E), another approximation for the normal distribution, is based on a series derived by Kerridge and Cook (1976). As in ZSTRECOK, our algorithm supplements the series with Peizer and Pratt's (1968) approximation for large values of $z$. The series is

$$
\int_{0}^{z} e^{-x^{2} / 2} d x=2 e^{-z^{2} / 8} \sum_{n=0}^{\infty}(z / 2)^{(2 n+1)} \frac{H_{2 n}(z / 2)}{(2 n+1) !},
$$

where $H_{n}(x)$ is the $n$th Hermite polynomial. Acknowledging the complicated nature of Equation 6, Kerridge and Cook showed how to simplify its computation by a transformation $\left[\theta_{n}(x)=x^{n} H_{n}(x) / n !\right]$ that reduces the magnitude of the numbers involved and by a recurrence relation for $\theta_{n}(x)$ [viz., $\theta_{n+1}=x^{2}\left(\theta_{n}-\theta_{n-1}\right) /(n+1)$, with $\theta_{0}(x)=1$ and $\left.\theta_{1}(x)=x^{2}\right]$ that corresponds to a standard recurrence relation for $H_{n}(x)$. The resulting series,

$$
\int_{0}^{z} e^{-x^{2} / 2} d x=z e^{-z^{2} / 8} \sum_{n=0}^{\infty} \frac{\theta_{2 n}(z / 2)}{(2 n+1)},
$$

in conjunction with the recurrence relation, affords surprisingly simple coding in a computer routine. An approximation to the normal distribution is obtained through summing enough terms in Equation 7 for convergence to the desired accuracy and then multiplying the result by $(2 \pi)^{-1 / 2}$. According to Moran (1980), the Kerridge and Cook series is not as accurate as his approximations, which have 9-place accuracy, but Kerridge and Cook reported 15-place accuracy with $z \leq 6$ using 30 or fewer terms of their series. ZKERCOOK is written to yield results as accurate as possible on the computer on which it is run. The number of terms needed is a direct function of $z$. With 16-digit arithmetic, between 4 and 37 terms are used for $z$ values between 0.01 and 6.8. ZKERCOOK requires input of $z$; it returns $A, p$, and the execution time.

The portions of ZSTRECOK and ZKERCOOK that are used with large values of $z$ approximate $p$ as

$$
(2 \pi)^{-1 / 2} e^{-z^{2} / 2} m /|z|
$$

where

$$
m=1-1 /\left\{z^{2}+3-1 /\left[0.22\left(z^{2}+3.2\right)\right]\right\} .
$$

Peizer and Pratt (1968) devised this approximation by adapting Laplace's continued fraction for the normal distribution (Zelen \& Severo, 1964, section 26.2.14). We tested a bound from Zelen and Severo (1964, section 26.2.24, $\mathrm{P}_{2}$ ) as an alternative approximation for large $z$ and found it to be a little less accurate than the Peizer and Pratt approximation. The crossover points between the two parts of ZSTRECOK and ZKERCOOK were determined empirically. The Peizer and Pratt approximation is used with ZSTRECOK when $z>6.9$; it is used with ZKERCOOK when $z>6.8$. In such cases, these programs calculate $p$ directly rather than as $(.5-A)$ and thus avoid the loss of precision that could otherwise occur.

\section{Tests of the Algorithms}

All tests were run on an IBM PC microcomputer with GW-BASICA version 3.2. The /D switch was used in invoking BASIC to enable double-precision evaluation of the exponential function, which is used in all algorithms, and the sine function, which is used in ZSTRECOK. With ZSIMPIT, $r$ was entered as 5E-16. The accuracy of the algorithms was assessed first in comparison with test values of $p$ from the tables of the National Bureau of Standards (1953), which provide 15-decimal-place values of $2 A$ for many values of $z$ through infinity, and 7-significantfigure values of $2 p$ for $z=6.00(0.01) 10.00$. Simple arithmetic manipulation of the tabled entries yielded the necessary values of $p$, which should be correct to at least 3 units in the 16th place. When the tables offered a choice of values for a given $z$, the more precise value was used. ZSIMPIT, ZSTRECOK, and ZKERCOOK were tested with $z=0.02(0.02) 10.00$. ZQUICK and ZQUICK.EXT were tested with the same values, except that the maximum $z$ was 6.5 for ZQUICK and 9.0 for ZQUICK.EXT. The algorithms also were tested with 17figure $z$ values rounded from White's (1970) tables, which provide 20-decimal-place $z$ values corresponding to $p=$ $.010(.005) .500$ and $p=10^{-m}, 2.5 \times 10^{-m}$, and $5.0 \times 10^{-m}$, where $m$ signifies all positive integers from 3 through 20 . ZSIMPIT, ZSTRECOK, and ZKERCOOK were tested with all 153 values in White; ZQUICK and ZQUICK.EXT were tested with all but the largest 24 and 4 values, respectively, because those large values exceed the scope of the algorithms.

Table 1 summarizes the results. All of the algorithms except ZQUICK appear a little more accurate in comparison with the more precise test values from White (1970), and those results are likely more valid than the comparisons with the tables of the National Bureau of Standards (1953). ZQUICK.EXT, ZSTRECOK, and ZKERCOOK are accurate to within 1 unit in the 16th decimal place. ZSIMPIT is accurate to within 2 units in the 16th place.

Further analysis of the accuracy of ZQUICK is necessary because the algorithm is most vulnerable in evaluating a $z$ value that is at the lower bound of a subinterval. Therefore, ZQUICK was tested with a number of $z$ values just greater than the lower bound of each subinterval. The maximum absolute error of ZQUICK was determined to be $4.9 \mathrm{E}-12$ (with $z$ values that exceed the lower bound

Table 1

Maximum Absolute Error of Algorithms

\begin{tabular}{lcr}
\hline & \multicolumn{2}{c}{ Source of Test Values } \\
\cline { 2 - 3 } \multicolumn{1}{c}{ Algorithm } & $\begin{array}{c}\text { National Bureau } \\
\text { of Standards }\end{array}$ & White \\
\hline ZSIMPIT* & $5.2 \mathrm{E}-16$ & $2.1 \mathrm{E}-16$ \\
ZQUICK $\dagger$ & $3.6 \mathrm{E}-12$ & $4.1 \mathrm{E}-12$ \\
ZQUICK.EXT & $2.8 \mathrm{E}-16$ & $6 \mathrm{E}-17$ \\
ZSTRECOK & $3.1 \mathrm{E}-16$ & $8 \mathrm{E}-17$ \\
ZKERCOOK & $3.1 \mathrm{E}-16$ & $8 \mathrm{E}-17$ \\
\hline
\end{tabular}

*Tested with "acceptable error" $=5 \mathrm{E}-16$. †Actual maximum absolute error $=4.9 \mathrm{E}-12$. 
of the subintervals by $1 E-14,1 E-15$, and $1 E-16$ ), although for most $z s$ the error is much less than that.

The above findings pertain to one-tailed $p$ and, of course, the error for two-tailed $p$ is twice as great. For two-tailed $p$, therefore, ZQUICK is accurate in all 10 decimal places that are displayed, ZSIMPIT is accurate to within 4 units in the 16th decimal place, ZQUICK.EXT to within 1 unit in the 16th place, and ZSTRECOK and ZKERCOOK to within 2 units in the 16th place. In an attempt to enhance these accuracies, we explored the impact of 17-place instead of 16-place constants in ZQUICK.EXT, ZSTRECOK, and ZKERCOOK and found that the 17-place constants did increase accuracy of the results by a few units in the 17 th decimal place. We have not, however, used the 17-place constants in the programs because of the uncertainty in introducing them when the computer monitors and program listings display only 16 digits.

Figure 1 shows representative execution times for ZQUICK.EXT, ZSTRECOK, and ZKERCOOK. Because the execution times of ZQUICK.EXT vary greatly with the distance of $z$ from the upper bound of the pertinent subinterval, the $z$ values for which times are shown were selected from the middle of the subintervals. For most $z$ values, ZKERCOOK is the fastest of these three highly accurate programs. Its time increases slightly with increasing $z$ through 6.8 because of the greater number of terms evaluated, with a maximum time of $1.2 \mathrm{sec}$. ZSTRECOK is next fastest, maintaining essentially the same time $(2.2-2.3 \mathrm{sec})$ for all $z$ values through 6.9 . In contrast to ZKERCOOK, ZQUICK.EXT tends to gain speed as $z$ increases, reflecting the decreasing $f^{\mathrm{iv}}(z)$ and the resulting decrease in the number of segments required to approximate the area. Although ZQUICK.EXT often is relatively slow, sometimes taking up to $40.7 \mathrm{sec}$, it executes faster than ZSTRECOK or ZKERCOOK when $z=6.75$. It loses the time advantage again with slightly larger $z$ values, for which ZSTRECOK and ZKERCOOK employ the fast Peizer and Pratt (1968) approximation, which executes in $0.1 \mathrm{sec}$. ZSIMPIT is exceptionally slow when $r$ is small, requiring up to $340 \mathrm{sec}$ to evaluate as many as 8,192 segments when $r=5 \mathrm{E}-16$. Even with $r=5 \mathrm{E}-11$, a setting that produces results as accurate as the target accuracy of ZQUICK, ZSIMPIT takes up to $21 \mathrm{sec}$. ZQUICK, on the other hand, evaluates all $z$ values in from 0.5 to $1.2 \mathrm{sec}$.

We used GW-BASICA instead of IBM Personal Computer BASIC to test the algorithms, because GW-BASICA is much faster. For example, the average times required to run ZQUICK.EXT, ZSTRECOK, and ZKERCOOK under GW-BASICA with the $z$ values shown in Figure 1 were only $46 \%, 66 \%$, and $37 \%$, respectively, of the times required under IBM BASIC version 3.1.

\section{Other Algorithms}

Many other algorithms have been developed to approximate the normal distribution (Johnson \& Kotz, 1970, chap. 13; Kennedy \& Gentle, 1980, chap. 5), and comparative evaluations of a number of them have been published (e.g., Brophy, 1983; Hill \& Joyce, 1967b; Kerridge \& Cook, 1976). Only a few of the better known or especially accurate algorithms are mentioned here. Ibbetson's (1963) algorithm is a classic that is sometimes recommended to obtain fast 7-decimal-place results (Hill, 1969; Hill \& Joyce, 1967b), although a simpler approximation of Zelen and Severo (1964, section 26.2.17), which was adapted from Hastings (1955), is almost as accurate. An algorithm of Milton and Hotchkiss (1969) is correct to

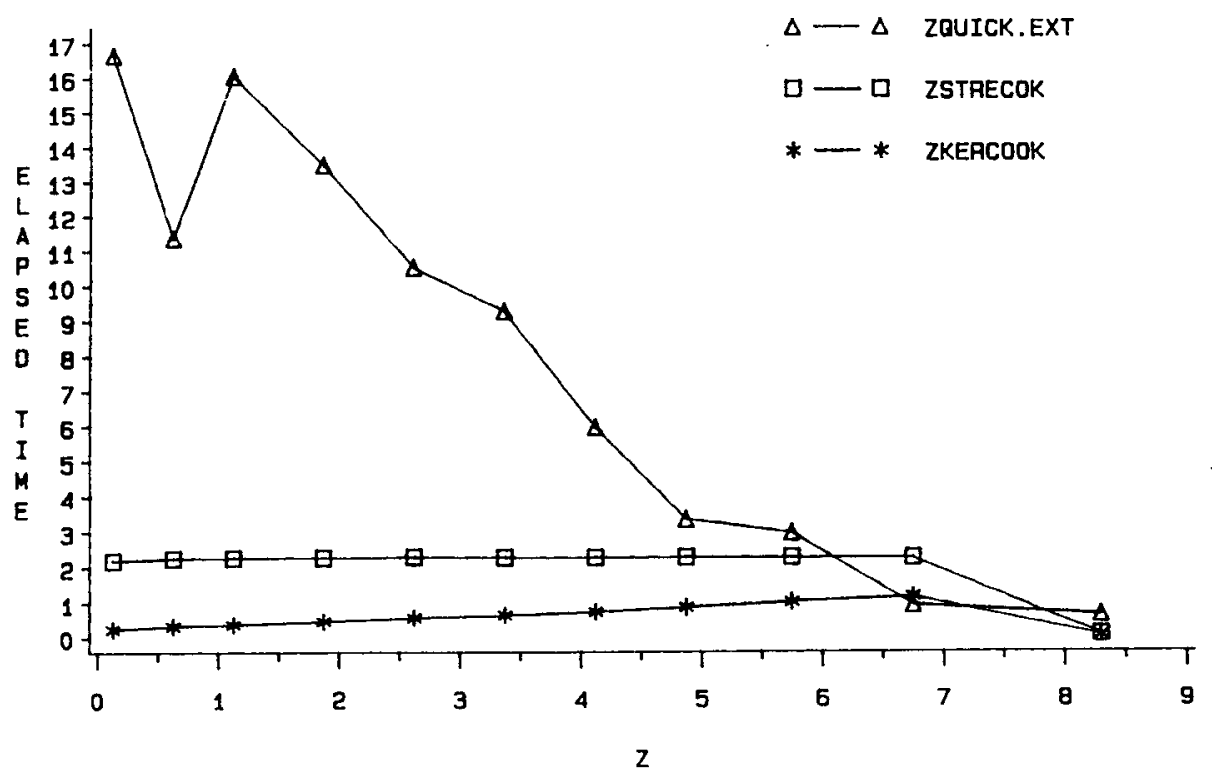

Figure 1. Execution time (in sec) of three normal curve programs as a function of $z$, run under GW-BASIC 3.2 on the IBM PC microcomputer. 
9 decimal places, as are two compact approximations that Moran (1980) derived from Strecok (1968). Algorithms of Adams (1969a) and Hill (1973), which are nearly identical, are accurate to 10 places. A relatively slow algorithm of Cooper (1968) offers similar accuracy, and it can be modified for greater accuracy (Hill, 1969).

Hill and Joyce (1967a) developed a highly accurate algorithm, comprising a power series (Zelen \& Severo, 1964, section 26.2.11) and a continued fraction (Zelen \& Severo, 1964, section 2.26.14) for small and large $z$ values, respectively. In fact, White (1970) used those methods in preparing his high-precision tables. The power series is rather frequently used by itself (e.g., Alf \& Grossberg, 1987; Dunlap \& Brown, 1983; Dunlap \& Myers, 1984), but it is not extremely accurate when $z$ is large. For example, on a computer with 16-digit arithmetic, Kerridge and Cook (1976) found that the power series could not attain 15-decimal-place accuracy when $z \geq 4$, or 10-decimal-place accuracy when $z=6$. However, the complete Hill and Joyce (1967a) algorithm is essentially exact to the accuracy of the computer (Hill \& Joyce, 1967b) and thus competitive with ZQUICK.EXT, ZSTRECOK, and ZKERCOOK; moreover, it is more accurate than those algorithms in evaluating $z$ values larger than about 2. Adams (1969b) and Holmgren (1970) have reported that the Hill and Joyce algorithm sometimes runs into overflow, which is never a problem with the algorithms presented here. Holmgren recommended changes to correct that, and Adams (1969b) and Bergson (1968) recommended other changes to increase the speed of the algorithm.

\section{Discussion}

The algorithms explicated in this paper have been tested thoroughly against some of the best tables available, and these tests have led us to some conclusions that users of $f(z)$ programs should know.

First, it is clear that the usefulness of Simpson's rule in normal curve work depends on the level of precision desired. Wood's (1987) program NORMLCV.QIK uses Simpson's rule to find probabilities for $f(z)$ rapidly with 6- to 9-place accuracy, and our ZQUICK always delivers 10 -place accuracy very quickly. Beyond 10 decimal places, Simpson's rule loses its efficiency, requiring that the program become long or slow or both. ZQUICK.EXT, for example, is longer than ZSTRECOK or ZKERCOOK and, with $z<6.75$, it is slower.

Our second observation is that $f(z)$ programs using Simpson's rule have the advantage of increasing speed as a function of $z$ because the fourth derivative rapidly diminishes as $z$ increases, which in turn reduces the size of $N$ returned by Inequality 2 . Although $f^{\text {iv }}(z)$ has its $\max$ imum value of 1.197 at $z=0, f^{\text {iv }}(z)$ drops rapidly, though not monotonically, to 0.484 at $z=1$, to 0.270 at $z=2$, to 0.133 at $z=3$, to 0.022 at $z=4$, and to 0.0007 at $z=5$. This explains the rule's strength in finding $f(z)$ areas when $z$ is large, whereas many other algorithms require help. Programs using Simpson's rule, which do not require add-on subroutines, are more straightforward and more readily comprehended than hybrid algorithms like ZSTRECOK and ZKERCOOK. Indeed, Kerridge and Cook (1976, p. 402) expressed this thought articulately: "It is a great advantage to be able to use a single formula in computing, instead of the usual two: see for example Hill \& Joyce (1967[a])."

Third, our tests of these programs have led us to recommend ZQUICK enthusiastically for these reasons: (1) Its accuracy is adequate for any task that behavioral researchers are likely to undertake. (2) It is quick, with running times in the 0.5 to $1.2 \mathrm{sec}$ range, and most runs are completed in about $1 \mathrm{sec}$ on the IBM PC. We use XT-compatible computers with 8- and $10-\mathrm{MHz}$ clocks, and when run on these machines, ZQUICK gives almost instant results, always consuming less than $0.5 \mathrm{sec}$. (3) ZQUICK is not long, and because almost all microcomputers now have ample memory and disk drives, program length is far less of a consideration than it was formerly.

Fourth, ZKERCOOK is a useful routine when greater accuracy or program brevity is desired, and it is relatively fast with most values of $z$. Although its algorithm is not as intuitively clear as that of ZQUICK, ZKERCOOK has applications in situations calling for high precision, when tables such as those of the National Bureau of Standards (1953) or Zelen and Severo (1964, Table 26.1) were formerly required.

Fifth, we would warn that highly respected probability tables contain some errors. We were brought to this depressing conclusion by the fact that for some zs (e.g., 2.20) ZSTRECOK, ZKERCOOK, and ZQUICK.EXT agree to 16 decimal places, although they disagree with the 15-place probability value given by Zelen and Severo (1964, Table 26.1). These algorithms are fundamentally different, approaching $f(z)$ from disparate perspectives, and one assumes that an accurate result has been obtained when they agree.

Finally, we are aware that primitive $f(z)$ routines are still in use simply out of habit. Now, people who use normal curve areas should abandon slow programs or those having uncertain accuracy for programs that are both very fast and highly accurate.

\section{Availability}

Listings of the programs, as shown in the Appendixes, can be obtained without charge from D. Louis Wood, University of Arkansas at Little Rock, 2801 South University, Little Rock, AR 72204. For a disk copy, send a formatted (MS/PC-DOS 2.0 or later) 5.25-in. (13.335-cm) disk and a self-addressed, postage-paid mailer.

\section{REFERENCES}

ADams, A. G. (1969a). Algorithm 39: Areas under the normal curve. Computer Joumal, 12, 197-198.

ADAMS, A. G. (1969b). Remark on Algorithm 304. Communications of the $A C M, 12,565-566$.

Alf, E. F., JR., Grossberg, J. M. (1987). DORF2R.BAS: Analyzing signal-detection theory rating data in the BASIC programming language. Behavior Research Methods, Instruments, \& Computers, $19,475-482$. 
Anton, H. (1981). Calculus. New York: Wiley.

Bergson, A. (1968). Certification of and remark on Algorithm 304. Communications of the $A C M, 11,271$.

Brophy, A. L. (1983). Accuracy and speed of seven approximations of the normal distribution function. Behavior Research Methods \& Instrumentation, 15, 604-605.

COOPER, B. E. (1968). Algorithm AS 2: The normal integral. Applied Statistics, 17, 186-188.

Cyvin, S. J. (1964). Algorithm 226: Normal distribution function. Communications of the $A C M, 7,295$.

Davis, P. J., \& RABINOWITz, P. (1975). Methods of numerical integration. New York: Academic Press.

DunlaP, W. P., \& Brown, S. G. (1983). FORTRAN IV functions to compute expected normal scores. Behavior Research Methods \& Instrumentation, 15, 395-397.

DUNLAY, W. P., MYERS, L. (1984). FORTRAN IV functions for computing expected half-normal scores. Behavior Research Methods, Instruments, \& Computers, 16, 401-404.

Hastings, C., JR. (1955). Approximations for digital computers. Princeton, NJ: Princeton University Press.

HiLL, I. D. (1969). Remark AS R2: A remark on Algorithm AS 2. Applied Statistics, 18, 229-300.

Hill, I. D. (1973). Algorithm AS 66: The normal integral. Applied Statistics, 22, 424-427.

Hill, I. D., \& JoYCE, S. A. (1967a). Algorithm 304: Normal curve integral. Communications of the ACM, 10, 374-375.

HiLl, I. D., \&oYCE, S. A. (1967b). Remarks on Algorithms 123, $180,181,209,226,272,304$. Communications of the ACM, 10, 377-378.

Holmgren, B. (1970). Remark on Algorithm 304. Communications of the $A C M, 13,624$.

IBBETSON, D. (1963). Algorithm 209: Gauss. Communications of the $A C M, 6,616$.

Johnson, N. L., Kotz, S. (1970). Distributions in statistics: Continuous univariate distributions - 1. Boston: Houghton Mifflin.

Kennedy, W., \& Gentle, J. (1980). Statistical computing. New York: Marcel Dekker.

Kerridge, D. F., \& Cook, G. W. (1976). Yet another series for the normal integral. Biometrika, 63, 401-403.

Milton, R. C., \& Hotchkiss, R. (1969). Computer evaluation of the normal and inverse normal distribution functions. Technometrics, 11 , 817-822.

Moran, P. A. P. (1980). Calculation of the normal distribution function. Biometrika, 67, 675-676.

National BureaU of Standards. (1953). Tables of nomal probability functions (Applied Mathematics Series 23). Washington, DC: U.S. Government Printing Office.

Pearson, E. S., Hartley, H. O. (Eds.) (1972). Biometrika tables for statisticians (Vol. 2). Cambridge, England: Cambridge University Press.

Peizer, D. B., \& Pratt, J. W. (1968). A normal approximation for binomial, F, beta, and other common, related tail probabilities, I. Journal of the American Statistical Association, 63, 1416-1456.

SMits, G. J. (1981). A FORTRAN IV function to compute the probability of a standard normal deviate. Behavior Research Methods \& Instrumentation, 13, 701.

STRECOK, A. J. (1968). On the calculation of the inverse of the error function. Mathematics of Computation, 22, 144-158.

WhITE, J. S. (1970). Tables of normal percentile points. Journal of the American Statistical Association, 65, 635-638.

Woon, D. L. (1985). High precision chi-square and normal curve integration. Behavior Research Methods, Instruments, \& Computers, 17, 429-431.

Wood, D. L. (1987). The last normal curve programs. Behavior Research Methods, Instruments, \& Computers, 19, 338-344.

Zelen, M., \& Severo, N. C. (1964). Probability functions. In M. Abramowitz \& I. A. Stegun (Eds.), Handbook of mathematical functions (pp. 925-995). Washington, DC: U.S. Government Printing Office.

\section{APPENDIX A \\ Listing of Program ZSIMPIT}

10 CLS: PRINT"ZSIMPIT": PRINT

20 DEFDBL C-H, P, S, X-Z: DEFINT I, N

$30 \times \mathrm{X}=0: \mathrm{S} 4=0: \mathrm{X}=0: \mathrm{H}=0 ; \mathrm{C}=-.5 \#$

40 INPUT"Enter z ", 2

50 INPUT"Acceptable error "; E

$60 \mathrm{VI}=\mathrm{TIMER}$

$70 \mathrm{H}=\mathrm{ABS}(Z) / 2: S 4=\mathrm{EXP}\left(\mathrm{H}^{\star} \mathrm{H}{ }^{\star} \mathrm{C}\right): \mathrm{S} 1=1+\mathrm{EXP}\left(Z^{\star} Z^{\star} \mathrm{C}\right): \mathrm{S} 2=0$

$80 \mathrm{~N}=2: \mathrm{Y}=.3989422804014327$ \# $: \mathrm{S}=\mathrm{Y} *(\mathrm{~S} 1+4 * \mathrm{~S} 4) * \mathrm{H} / 3$

$90 \mathrm{~S} 2=\mathrm{S} 2+\mathrm{S} 4: \quad \mathrm{S} 4=0: \quad \mathrm{X}=\mathrm{H} / 2$

100 FOR I=0 TO N-1: XI=X+I*H: $54=\mathrm{S} 4+\mathrm{EXP}(\mathrm{X} 1 * \mathrm{X} 1 * \mathrm{C}):$ NEXT I

$110 \mathrm{SS}=\mathrm{Y} *(\mathrm{~S} 1+4 * \mathrm{~S} 4+2 * \mathrm{~S} 2) * \mathrm{X} / 3: \mathrm{N}=\mathrm{N}+\mathrm{N}$ : IF ABS $(\mathrm{S}-\mathrm{SS})<\mathrm{E}$ THEN 130

$120 \mathrm{~S}=\mathrm{SS}: \mathrm{H}=\mathrm{X}$ : GOTO 90

$130 \mathrm{EE}=\mathrm{ABS}(\mathrm{S}-\mathrm{SS}):$ IF SS>.5\# THEN SS $=.5$ \#

$140 \mathrm{~V} 2=$ T IMER

150 PRINT

160 PRINT"Area $(0-z)="$ SS

$170 \mathrm{P}=5$. - -SS: PRINT"p (one-tailed) $=" \mathrm{P}$

180 PRINT

190 PRINT"Estimated error $<="$ EE

200 PRINT"Number of segments $=" \mathrm{~N}$

210 PRINT

220 PRINT"El apsed Time =" USING" \#\#\#\#.\#\# sec"; V2-VL

230 END

\section{APPENDIX B Listing of Program ZQUICK}

1 CLS

2 PRINT"This Program Computes Exact Normal Curve Probabilities "

3 FOR I $=1$ TO 2000: NEXT

4 CLEAR:DEFDBL A-Z:DEFINT J,M:DEFSNG V

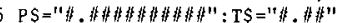

6 CLS: PRINT"ZQUICK" : PRINT

7 INPUT"Enter $z$ ", L

$8 \mathrm{~V} \mathrm{I}=\mathrm{T}$ IMER

$9 \mathrm{~L}=\mathrm{ABS}(\mathrm{L}):$ IF $\mathrm{L}<.0000000001$ / THEN $\mathrm{AA}=0$ f: $: \mathrm{A}=.5$ \#: GOTO 17

10 GOSUB 29

$11 \mathrm{Q}=(\mathrm{U}-\mathrm{L}) /(\mathrm{M} \star 2)$

$12 \mathrm{X}=\mathrm{L}: \operatorname{COSUB} 28: \mathrm{S}=\mathrm{S}+\mathrm{Y}: \mathrm{X}=\mathrm{U}: \operatorname{GOSUB} \quad 28: \mathrm{S}=\mathrm{S}+\mathrm{Y}$

13 FOR J $=1$ TO $M: X=L+(2 \star J-1) \star Q: \operatorname{COSUB} 28: T=T+Y: N E X T$

$14 \mathrm{~S}=\mathrm{S}+4 * \mathrm{~T}: \mathrm{Y}=\mathrm{M}-\mathrm{I}: \mathrm{T}=0$

15 FOR J=1 TO $M: X=L+2 * J * Q: \operatorname{GOSUB} 28 ; \mathrm{T}=\mathrm{T}+\mathrm{Y}: \mathrm{NEXT}$

$16 \mathrm{~S}=\mathrm{S}+2 * \mathrm{~T}: \mathrm{A}=\mathrm{S} * \mathrm{Q} / 3+\mathrm{AREA}: \mathrm{AA}=.5 / / \mathrm{A}$

17 V $2=$ TIMER

18 PRINT"The One-tailed Probability of $z$ is "USING PS;A

19 PRINT

20 PRINT"The Area From 0 To $z$ Is "USING P\$;AA;

21 PRINT TAB(39)"The Area Between $-z$ And $z$ Is "USING P\$; $2 * A A$

22 PRINT

23 PRINT"The Two-tailed Probability Is "USING P\$; $2^{\star A}$

24 PRINT

25 PRINT"Elapsed Time="USING T\$; V2-V1;:PRINT" sec"

26 PRINT:PRINT"For Another Problem Press The Spacebar ": PRINT

27 ZS=INKEYS:IF Z\$="'"THEN 27 ELSE 4

$28 \mathrm{Y}=.3989422804014327$ \#/EXP $(X \star X / 2): R E T U R N$

29 IF L>6.5\# THEN PRINT: PRINT"One-tailed $P<.000000000 \mathrm{I}^{\prime \prime}$ : PRINT : END

30 PRINT"C O M P U T I N G !"

31 IF $\mathrm{L}>2.5$ \#HEN 45

32 IF $L<=.17$ \# THEN $M=11: U=.17$ \#: AREA $=.4325050683249616$ \#: RETURN

33 IF $L<=.34$ \# THEN $M=10: U=.34$ \# $:$ AREA $=.3669282639639719$ \#: RETURN

34 IF $\mathrm{L}<=.52$ \# THEN $M=10: U=.52 \|$ : AREA $=.3015317875469662$ \#: RETURN

35 IF $L<=.72$ \# THEN $M=10: U=.72 \#:$ AREA $=.2357624977792512$ \#: RETURN

36 IF $L<=.93$ \# THEN $M=10: U=.93$ \#:AREA $=.1761855422452579$ \#: RETURN

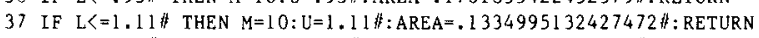

38 IF $\mathrm{L}<=1$.3 \# THEN $M=11: \mathrm{U}=1.3$ \#: AREA $=.0968004845856103$ \#: RETURN

39 IF $\mathrm{L}<=1.48$ \# THEN $\mathrm{M}=11: \mathrm{U}=1.48$ \#: AREA $=.0694366233333317$ \#: RETURN

40 If $L<=1.67$ \#HEN $M=11: U=1.67$ \#:AREA $=.0474596818029473$ \#:RETURN

41 IF $L<=1.86$ \#HEN $M=11: U=1.86$ \#: $A R E A=.0314427629807527 \#:$ RETURN

42 IF $L<=2.05$ \# THEN $M=9: U=2.05$ \#: AREA $=.0201822154057044$ \#: RETURN

43 IF $\mathrm{L}<=2.25$ \# THEN $M=9: U=2.25$ \#: $A$ AREA $=.0122244726550447$ \#: $R E T U R N$

44 IF $\mathrm{L}<=2.5$ \#HEN $M=9: \mathrm{U}=2.5$ \#: $\mathrm{AREA}=.0062096653257762$ \#: RETURN

45 IF $\mathrm{L}<=2.7$ \# THEN $M=8: U=2.7$ \#: AREA $=.0034669738030406$ \# : RETURN

46 IF $\mathrm{L}<=2.9$ \# THEN $M=8: \mathrm{U}=2.9$ \# $:$ AREA $=.001865813300384$ \#: RETURN

47 IF $L<=3.1$ \# THEN $M=8: U=3.1$ \#: AREA $=.0009676032132183$ \#: RETURN

48 IF $\mathrm{L}\langle=3.3$ H THEN $M=8: U=3.3$ \#:AREA $=.0004834241423838$ \#: RETURN

49 IF $L<=3.5$ \#HEN $M=7: U=3.5$ \#: AREA $=.0002326290790355 \%:$ RETURN

50 IF $L<=3.7$ \# THEN $M=7: U=3.7$ \#: $A R E A=.0001077997334774$ औ: RETURN

51 IF $L<=3.9$ i/ THEN $M=6: U=3.9$ 传: $A R E A=.0000480963440176$ / : RETURN

52 IF $L<=4.1$ \# THEN $M=6: U=4.1$ \#: $A$ REA $=.0000206575069125$ \#: RETURN

53 IF $\mathrm{L}<=4.35$ \# THEN $M=6: U=4.35$ \# : $A R E A=.0000068068765993$ \#: $R E$ TURN

54 IF $L<=4.6 \#$ THEN $M=5: U=4.6 \#:$ AREA $=.0000021124547025 \#:$ RETURN

55 IF $\mathrm{L}<=4.9$ \# THEN $M=5: U=4.9 \sharp: A R E A=.0000004791832766$ \#: RETURN

56 IF $L<=5.3 \#$ THEN $M=5: U=5.3 \#:$ AREA $=.0000000579013404 \#:$ KETURN

57 IF $L<=5.8$ \# THEN $M=4: U=5.8$ \#: $A$ REA $=.000000003315746$ \#: RETURN

$58 \mathrm{M}=4: U=6.5 \#:$ AREA $=.00000000004016 \|:$ RETURN 


\section{APPENDIX C \\ Listing of Program ZQUICK.EXT}

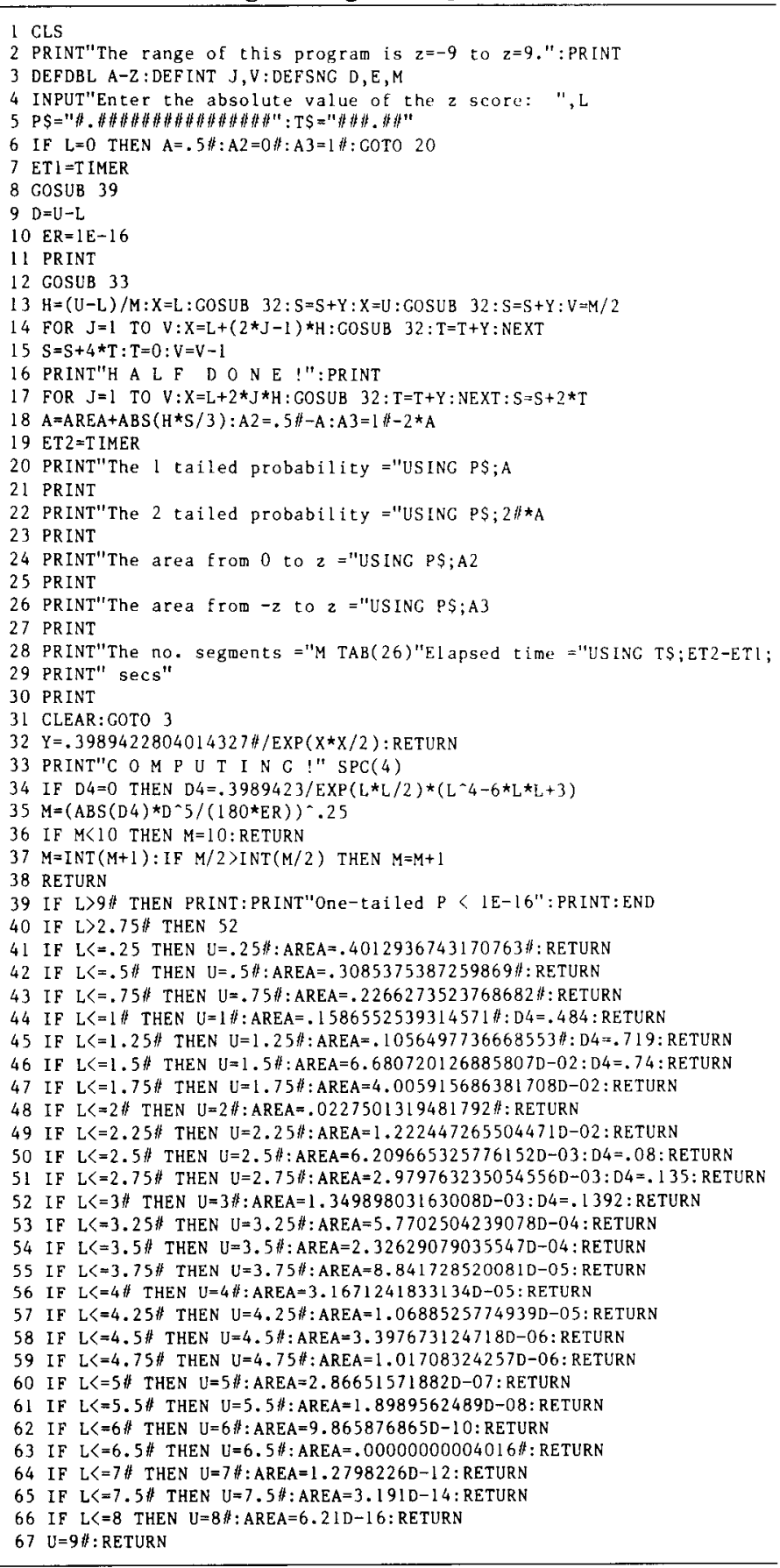

\section{APPENDIX D \\ Listing of Program ZSTRECOK}

10 CLS: PRINT"ZSTRECOK": PRINT

20 DEFDBL $P, W-2$ : DEFINT I

30 INPUT"Enter z ", 2

$40 \mathrm{Vl}=\mathrm{T}$ IMER

$50 \mathrm{Z}=\mathrm{ABS}(\mathrm{Z})$

60 IF $Z>6.9$ \# THEN $Z Z=Z \star Z: W=1-1 /(Z Z+3-1 /(.22 \# \star Z Z+.704 \|)):$ $\mathrm{Pl}=.3989422804014327$ \#*EXP $(-\mathrm{ZZ} / 2) * \mathrm{~W} / \mathrm{Z}: \mathrm{P}=.5$ \# $-\mathrm{P} 1:$ COTO 100

$70 \mathrm{P}=.1414213562373095 / \approx \approx \mathrm{Z}: \mathrm{X}=\mathrm{P}+\mathrm{P}: \quad \mathrm{Y}=-.04 \pi$

80 FOR I $=1$ TO 29: $P=P+E X P(I * I * Y) / I * \operatorname{SIN}(I * X): \operatorname{NEXT~I~}$

$90 \mathrm{P}=.3183098861837907$ / $* \mathrm{P}: \mathrm{Pl}=.5$ औ $-\mathrm{P}$
100 V $2=T$ IMER

110 PRINT

120 PRINT"Area $(0-z)=" P$

130 PRINT"P (one-tailed) =" PI

140 PRINT

150 PRINT"El apsed Tíme ="; USING" \#.\# sec"; V2-V1

160 END

$170 \mathrm{REM}: .3989422804014327=1 / \mathrm{SQR}\left(2 \star^{*} \mathrm{PI}\right) \quad($ Line 60$)$

$180-.1414213562373095=1 /(\operatorname{SQR}(2) * 5) \quad($ Line 70$)$

$190-.3183098861837907=1 /$ PI $\quad$ (Line 90) 
APPENDIX E

Listing of Program ZKERCOOK

\author{
10 CLS: PRINT"ZKERCOOK": PRINT \\ 20 DEFDBL P, T, W, Z: DEFINT I \\ 30 INPUT"Enter $z ", Z$ \\ $40 \mathrm{Vl}=$ = IMER \\ $50 Z=A B S(Z): \quad P S=.3989422804014327$ \\ 60 IF $Z>6.8 \#$ THEN $Z Z=Z * Z: W=1-1 /(Z Z+3-1 /(.22 \# \star Z Z+.704 \#)):$
$\mathrm{Pl}=\mathrm{PS} * \mathrm{EXP}(-\mathrm{ZZ} / 2) \star W / Z: P=.5 \#-\mathrm{P} 1: \operatorname{GOTO}(10$ \\ $70 \mathrm{I}=2: \mathrm{T}=1: \quad \mathrm{P}=1 ; \mathrm{Tl}=2 * 2 / 4: \quad \mathrm{Zl}=\mathrm{T} 1: \mathrm{P} 1=0$ \\ $80 \mathrm{~T}=\mathrm{Z} 1 *(\mathrm{~T} 1-\mathrm{T}) / \mathrm{I}: \mathrm{I}=\mathrm{I}+1: \mathrm{P}=\mathrm{P}+\mathrm{T} / \mathrm{I}:$ IF $\mathrm{P}=\mathrm{Pl}$ THEN 100 \\ $90 \mathrm{~T} 1=\mathrm{Z} I^{\star}(\mathrm{T}-\mathrm{T} 1) / \mathrm{I}: \mathrm{I}=\mathrm{I}+\mathrm{L}: \mathrm{Pl}=\mathrm{P}:$ GOTO 80
}

(Manuscript received February 7, 1989; revision accepted for publication May 24, 1989.) 